\title{
Liouville Energy on a Topological Two Sphere
}

\author{
XiuXiong Chen • Meijun Zhu
}

Received: 11 April 2013 / Accepted: 20 November 2013 / Published online: 31 December 2013

(C) School of Mathematical Sciences, University of Science and Technology of China and Springer-Verlag Berlin Heidelberg 2013

\begin{abstract}
In this paper we shall give an analytic proof of the fact that the Liouville energy on a topological two sphere is bounded from below. Our proof does not rely on the uniformization theorem and the Onofri inequality, thus it is essentially needed in the alternative proof of the uniformization theorem via the Calabi flow. Such an analytic approach also sheds light on how to obtain the boundedness for $E_{1}$ energy in the study of general Kähler manifolds.
\end{abstract}

Keywords Uniformization theorem · Liouville energy · Moser-Trudinger-Onofri inequality · Blowup analysis

Mathematics Subject Classification (2010) 35A23 • 42B37

\section{Introduction}

\subsection{Description of the Problem}

Let $(M, g)$ be a smooth Riemann surface. For any conformal new metric $g_{1}=e^{u} g$, the corresponding Liouville energy is defined by

$$
L_{g}\left(g_{1}\right)=\int_{M} \ln \frac{g_{1}}{g} \cdot\left(R_{g_{1}} d V_{g_{1}}+R_{g} d V_{g}\right)
$$

where $R_{g}$ and $R_{g_{1}}$ are twice the Gaussian curvatures $K_{g}$ and $K_{g_{1}}$ with respect to metrics $g$ and $g_{1}$. Since

$$
R_{g_{1}}=e^{-u}\left(-\Delta_{g} u+R_{g}\right)
$$

X.X. Chen $(\varangle)$

Department of Mathematics, University of Wisconsin-Madison, Madison, WI 53706-1388, USA e-mail: xiuxiong.chen@gmail.com

M. Zhu

Department of Mathematics, The University of Oklahoma, Norman, OK 73019, USA 
the Liouville energy of metric $g_{1}$ can also be represented by

$$
L_{g}\left(g_{1}\right)=\int_{M}\left(\left|\nabla_{g} u\right|^{2}+2 R_{g} u\right) d V_{g} .
$$

It was showed by Chow [14] and Chen [7] that Ricci flow and Calabi flow can be viewed as the gradient flow of the Liouville energy. Therefore if $M$ is a topological two sphere, it is important to know in the study of Ricci and Calabi flow that the Liouville energy is bounded from below for any metric with fixed volume. In fact such boundedness can be used to show the global existence of Calabi flow via integral estimates (see, for example, Chen [7]), thus one can give an alternative proof of the uniformization theorem (for the hardest case: positive curvature case) via Calabi flow. ${ }^{1}$ However, present argument in the literature seems to be a tautology: the proof of the fact that the Liouville energy on a two sphere is bounded from below relies on the uniformization theorem. In fact, the proof can be summarized as follows. Let $(M, g)$ be a topological sphere. From the uniformization theorem we know that $(M, g)$ is conformally equivalent to the standard sphere $\left(S^{2}, g_{0}\right)$, that is, there is a $\varphi(x) \in C^{2}\left(S^{2}\right)$ such that $g=e^{\varphi} g_{0}$. For a given metric $g_{1}=e^{u} g$, since $g_{1}=e^{u+\varphi} g_{0}$ and $R_{g_{0}}=2$, we have

$$
\begin{aligned}
L_{g}\left(g_{1}\right) & =\int_{M}\left(\left|\nabla_{g} u\right|^{2}+2 R_{g} u\right) d V_{g} \\
& =L_{g_{0}}\left(g_{1}\right)-L_{g_{0}}(g) \\
& =\int_{S^{2}}\left(\left|\nabla_{g_{0}}(u+\varphi)\right|^{2}+4(u+\varphi)\right) d V_{g_{0}}-L_{g_{0}}(g) \\
& \geq 16 \pi \cdot \ln \frac{1}{4 \pi} \int_{S^{2}} e^{(u+\varphi)} d V_{g_{0}}-L_{g_{0}}(g) .
\end{aligned}
$$

The last inequality follows from the well-known Onofri inequality on $S^{2}$. Thus the finiteness of the volume of the metric $g_{1}$ implies that the Liouville energy of such metric is bounded from below.

\subsection{Historic Note}

It is always natural to ask if one can use variational approach to attack the existence of constant scalar curvature metrics in certain conformal class in 2-sphere. The elliptic approach might start with Berger [4], which eventually leads to the famous prescribing curvature problem on $S^{2}$ (the Nirenberg problem). The parabolic approach was first studied by Hamilton [17] and Chow [14] via Ricci flow, and later details of the approach via the Calabi flow were illustrated by the first author. The first step of such an approach would be to find a direct analytic proof of the fact the Liouville energy is bounded from below first. This question was raised in [7] as a key step in deriving a

\footnotetext{
${ }^{1}$ In a recent note, Chen, Lu and Tian [11] clarify that the Ricci flow did yield another proof of the uniformization theorem for spheres even without using current integral estimates. Nevertheless our result not only answer a conjecture of Chen [7], but also has broad applications in the study of Kähler manifolds (see the historic note).
} 
new proof for uniformization theorem in topological two sphere via the Calabi flow. More importantly, this question is closely related to a far reaching program in the study of general Kähler manifolds: Can one use variational approach to derive the existence of constant scalar curvature metrics in each Kähler class?

The search of constant scalar curvature metric in each Kähler class was suggested by Calabi [6] in 1982. This is one of the key problems in Kähler geometry and there is extensive research in this and related problems. The analytic obstruction to the existence of such metric was first observed in the important work of Mabuchi-Bando [3] in 1987, where they showed that the existence of Kähler Einstein metric implies the existence of lower bound of the K energy in each Kähler class. ${ }^{2}$ This obstruction was extended by the work of the first author in 1998 to the case of constant scalar curvature metric in Kähler manifold with non-positive first Chern class. More recently, this problem is completely settled for all Kähler classes (cf. [10] for more references). In [9], Chen and Tian introduced a set of $n+1$ energy functionals $E_{0}, E_{1}, \ldots, E_{n}$ in any $n$-dimensional Kähler manifold where $E_{0}$ is the $\mathrm{K}$ energy functional and $E_{1}$ is the Liouville energy on $S^{2}$. A natural question asked in [7] (Question 7.3) and [8] is whether the existence of a Kähler Einstein metric implies the lower bound of $E_{1}$. Concentrated effort by various geometers and rapid progress are made in this problem. For instance, the first author observed that, along the Kähler Ricci flow on Kähler Einstein manifold, this functional is bounded below. This is answered affirmatively by Song and Weinkove [24] that lower bound of $E_{1}$ is obstruction to the existence of Kähler Einstein metrics. Shortly after, Pali [22] gives a new proof which considerably simplified the Song-Weinkove's argument. See also [25] for other related results. All of these recent results highlight the importance of obtaining an a priori lower bound of this functional $E_{1}$ (without the knowledge of Kähler Einstein metric or constant scalar curvature metric). Unfortunately, even in the simplest case of $C P^{n}(n>1)$, we even do not know how to prove directly why $E_{1}$ energy is bounded from below without using Mabuchi-Bando theorem. On $S^{2}$, the previous proof (for example, given by Osgood-Phillips-Sarnak [23]) relies on the existence of constant scalar curvature metric (as we showed before). The present paper gives an a priori lower bound of $E_{1}$ (or Liouville energy) on the topological $S^{2}$. This certainly sheds light on how to analytically derive the low bound of the $E_{1}$ in higher dimension and it gives us hope that the large program can be successful.

\subsection{The Main Results}

The main purpose of this note is to give such an analytic proof of

Theorem 1.1 If $(M, g)$ is a topological two sphere with volume $4 \pi$ and bounded curvature, then there is a constant $C_{\text {low }}$ (which depends on metric $g$ ) such that for any conformal metric $g_{1}=e^{2 u} g$ with volume $4 \pi$,

$$
L\left(g_{1}\right) \geq C_{\text {low }}
$$

\footnotetext{
${ }^{2}$ On $S^{2}$, a Kähler class is equivalent a pointwise conformal class of Riemannian metric with fixed total area.
} 
Remark 1.1 The previous proof based on the uniformization theorem and the Onofri inequality does yield the best constant for $C_{\text {low }}$. Our proof cannot yield the best lower bound.

Comparing with some existing results for higher dimensional Yamabe type problem (see, for example, [18] and [19]), our proof seems quite standard. We start with a local sharp inequality (caution: this local inequality does not rely on Moser's inequality), which allows us to obtain a rough sharp inequality (or, sometimes, we refer it as an $\epsilon$-level inequality) in Proposition 2.1. We then study the behavior of the minimizing sequence as the parameter $\epsilon$ goes to 0 . It will be shown that the perturbed Liouville energy will stay uniformly bounded as $\epsilon$ goes to 0 , and this yields the result in Theorem 1.1. It worths of mentioning that in higher dimensional case, similar argument was carried out by Hebey and Vaugon [18] in their proof of a conjecture due to Aubin [1], and by Li and Zhu in the proof of sharp trace inequality [19].

\section{Preliminary Results}

We start with a local sharp inequality which is proved in [20]. It is interesting to point out that such a local inequality also yields a much simpler proof of the Onofri inequality (see also a more recent work [21]).

Let $\Omega \subset R^{2}$ be a bounded smooth domain, $\Omega^{*}$ be the ball in $R^{2}$ which has the same area as $\Omega$, and denote

$$
D_{a, b}(\Omega)=\left\{f(x)-b \in H_{0}^{1}(\Omega): \int_{\Omega} e^{2 f} d x=a\right\} .
$$

We have the following sharp inequality.

Theorem 2.1 [20]

$$
\inf _{w \in D_{a, b}(\Omega)} \int_{\Omega}|\nabla w|^{2} d x \geq 4 \pi \cdot\left(\ln \frac{a e^{-2 b}}{\pi r^{2}}+\frac{\pi r^{2}}{a e^{-2 b}}-1\right),
$$

where $r$ is the radius of $\Omega^{*}$.

As simple consequences, we have

Corollary 2.1 Let $\Omega \subset R^{2}$ be a smooth bounded domain. For any $u \in H_{0}^{1}(\Omega)$,

$$
\int_{\Omega} e^{u} d x \leq \pi r^{2} e \exp \left\{\frac{1}{16 \pi} \int_{\Omega}|\nabla u|^{2} d x\right\}
$$

where $r$ is the radius of $\Omega^{*}$.

And 
Corollary 2.2 Let $(M, g)$ be a smooth Riemann surface. For any given $\epsilon>0$, there are $r_{0}>0$ and $C(\epsilon)>0$ such that if $\Omega \subset B\left(x_{0}, r_{0}\right)$ for some point $x_{0} \in M$, then

$$
\int_{\Omega} e^{w} d V_{g} \leq C(\epsilon) \exp \left\{\left(\frac{1}{16 \pi}+\epsilon\right) \int_{\Omega}\left|\nabla_{g} w\right|^{2} d V_{g}\right\} \quad \forall w \in H_{0}^{1}(\Omega) .
$$

Remark 2.1 Inequalities in Corollaries 2.1 and 2.2 were proved early by Cherrier [13] based on Moser's inequality.

From these two corollaries we can derive

Lemma 2.1 Let $(M, g)$ be a given Riemann surface. For any given $\epsilon>0$, there are constants $C_{1}=C_{1}(\epsilon), C_{2}=C_{2}(\epsilon)$ such that

$$
\int_{M} e^{w} d V_{g} \leq C_{1} \exp \left\{\left(\frac{1}{16 \pi}+\epsilon\right) \int_{M}\left|\nabla_{g} w\right|^{2} d V_{g}+C_{2} \int_{M} w^{2}\right\}, \quad \forall w \in H^{1}(M) .
$$

Proof Let $r_{0}$ be the constant given in Corollary 2.2. Let $\bigcup_{i=1}^{N} B\left(r_{i} / 2, x_{i}\right)$ be a covering of $M$, where $r_{i}<r_{0}$. Let $\left\{\psi_{i}(x)\right\}_{i=1}^{N}$ be smooth cutoff functions subordinate to this covering, satisfying

$$
\psi_{i}(x)= \begin{cases}1, & x \in B\left(x_{i}, r_{i} / 2\right) \\ 0, & x \notin B\left(x_{i}, r_{i}\right)\end{cases}
$$

and $0 \leq \psi_{i}^{2} \leq 1$. We have, using Corollary 2.2,

$$
\begin{aligned}
\int_{M} e^{u} d V_{g} & \leq \sum_{i=1}^{N} \int_{B\left(x_{i}, r_{i} / 2\right)} e^{u} d V_{g} \\
& \leq \sum_{i=1}^{N} \int_{B\left(x_{i}, r_{i}\right)} e^{u \cdot \psi_{i}} d V_{g} \\
& \leq \sum_{i=1}^{N} C(\epsilon) \exp \left\{\left(\frac{1}{16 \pi}+\epsilon\right) \int_{B\left(x_{i}, r_{i}\right)}\left|\nabla_{g}\left(u \cdot \psi_{i}\right)\right|^{2} d V_{g}\right\}
\end{aligned}
$$

Note

$$
\begin{aligned}
\int_{B\left(x_{i}, r_{i}\right)}\left|\nabla_{g}\left(u \cdot \psi_{i}\right)\right|^{2} d V_{g} & \leq \int_{B\left(x_{i}, r_{i}\right)}\left(\left|\nabla_{g} u\right|^{2} \cdot\left|\psi_{i}\right|^{2}+C_{2} u^{2}\right) d V_{g} \\
& \leq \int_{M}\left(\left|\nabla_{g} u\right|^{2}+C_{2} u^{2}\right) d V_{g} .
\end{aligned}
$$

It follows that

$$
\int_{M} e^{u} d V_{g} \leq N \cdot C(\epsilon) \exp \left\{\left(\frac{1}{16 \pi}+\epsilon\right) \int_{M}\left(\left|\nabla_{g} u\right|^{2}+C_{2} u^{2}\right) d V_{g}\right\} .
$$


We are now ready to establish the following $\epsilon$-level inequality according to Aubin [2]. Similar type inequalities were discussed for higher dimensional cases. See, for example, [18] or [19].

Proposition 2.1 Let $(M, g)$ be a topological two sphere with bounded Gaussian curvature $K_{g}$. Then for any given $\epsilon>0$, there is a constant $C_{3}=C_{3}(\epsilon)$ such that for any $u \in H^{1}(M)$ with $\int_{M} K_{g} \cdot u d V_{g}=0$,

$$
\int_{M} e^{u} d V_{g} \leq C_{3}(\epsilon) \exp \left\{\left(\frac{1}{16 \pi}+\epsilon\right) \int_{M}\left|\nabla_{g} u\right|^{2} d V_{g}\right\} .
$$

To prove this proposition, we need the following Poincaré-Sobolev type inequality.

Lemma 2.2 Let $(M, g)$ be a topological two sphere with bounded Gaussian curvature $K_{g}$. Then for any $p \geq 1$, there is a constant $c_{p}$ such that for any $u \in H^{1}(M)$ with $\int_{M} K_{g} \cdot u d V_{g}=0$,

$$
\left(\int_{M}|u|^{p}\right)^{2 / p} \leq c_{p} \int_{M}\left|\nabla_{g} u\right|^{2} d V_{g}
$$

Proof The proof is standard. We prove it by contradiction. Suppose this is not true, then there is a sequence of functions $\left\{u_{n}\right\}$ in $H^{1}(M)$ with $\int_{M} K_{g} \cdot u_{n} d V_{g}=0$ such that

$$
\left(\int_{M}\left|u_{n}\right|^{p}\right)^{2 / p} \geq n \int_{M}\left|\nabla_{g} u_{n}\right|^{2} d V_{g}
$$

Let

$$
v_{n}=\frac{u_{n}}{\left(\int_{M}\left|u_{n}\right|^{p}\right)^{1 / p}} .
$$

We know that

$$
\left\|v_{n}\right\|_{L^{p}}=1, \quad\left\|v_{n}\right\|_{H^{1}} \leq C \quad \text { and } \quad\left\|\nabla_{g} v_{n}\right\|_{L^{2}} \rightarrow 0
$$

Thus, $v_{n} \rightarrow v_{0}$ in $H^{1}(M)$ and $v_{0}$ is a constant. By the compact embedding, we know that $v_{n} \rightarrow v_{0}$ in $L^{q}$ for any $q<\infty$, thus $\int_{M} K_{g} v_{0} d V_{g}=0$ and $\left\|v_{0}\right\|_{L^{p}}=1$. On the other hand, since $\int_{M} K_{g} d V_{g}=4 \pi$, we conclude that $v_{0}=0$. Contradiction.

Remark 2.2 The topological assumption is obviously needed since the above inequality does not hold for a flat torus.

Remark 2.3 The condition on $K_{g}$ could be relaxed to $\left\|K_{g}\right\|_{L^{q}(M)} \leq C$ for some $q>1$. However, it is not obvious whether the result is still true or not by only assuming that $K_{g} \in L^{1}(M)$.

We now return to the proof of Proposition 2.1. 
Proof of Proposition 2.1 Due to Lemma 1 in [1, p. 586], we only need to prove the proposition for functions in $C^{1}(M)$ with only non-degenerate critical points. Let $u(x)$ be such a function with $\int_{M} K_{g} \cdot u d V_{g}=0$. For small enough $\eta>0$, there exists $a_{\eta}$ such that

$$
\operatorname{Vol}\left\{x \in M: u(x)>a_{\eta}\right\}=\eta \text {. }
$$

Using Lemma 2.1 we have for any $\epsilon_{1}>0$, there are constants $C_{1}\left(\epsilon_{1}\right), C_{2}\left(\epsilon_{1}\right)>0$ such that

$$
\begin{aligned}
\int_{M} e^{u} d V_{g} \leq & e^{a_{\eta}} \cdot \int_{M} e^{\left(u-a_{\eta}\right)} d V_{g} \leq e^{a_{\eta}} \cdot \int_{M} e^{\left(u-a_{\eta}\right)_{+}} d V_{g} \\
\leq & e^{a_{\eta}} \cdot C_{1}\left(\epsilon_{1}\right) \exp \left\{\left(\frac{1}{16 \pi}+\epsilon_{1}\right) \int_{M}\left|\nabla_{g}\left(u-a_{\eta}\right)_{+}\right|^{2} d V_{g}\right. \\
& \left.+C_{2}\left(\epsilon_{1}\right) \int_{M}\left|\left(u-a_{\eta}\right)_{+}\right|^{2} d V_{g}\right\},
\end{aligned}
$$

where $f_{+}(x)=\max \{f(x), 0\}$. Since

$$
a_{\eta} \cdot \eta \leq \int_{\left\{x \in M: u(x)>a_{\eta}\right\}} u d V_{g} \leq \int_{M}|u| d V_{g} \leq c_{1}^{1 / 2}\left(\int_{M}\left|\nabla_{g} u\right|^{2}\right)^{1 / 2},
$$

we have

$$
a_{\eta} \leq \eta\left\|\nabla_{g} u\right\|_{L^{2}}^{2}+\frac{C}{\eta^{3}} .
$$

Also, using Lemma 2.2, we have

$$
\begin{aligned}
\int_{M}\left|\left(u-a_{\eta}\right)_{+}\right|^{2} d V_{g} & =\int_{\left\{x \in M: u(x)>a_{\eta}\right\}}\left|\left(u-a_{\eta}\right)_{+}\right|^{2} d V_{g} \\
& \leq\left(\int_{\left\{x \in M: u(x)>a_{\eta}\right\}}\left|\left(u-a_{\eta}\right)_{+}\right|^{4} d V_{g}\right)^{1 / 2} \cdot \eta^{1 / 2} \\
& \leq\left(\int_{M}|u|^{4} d V_{g}\right)^{1 / 2} \cdot \eta^{1 / 2} \\
& \leq c_{4}\left\|\nabla_{g} u\right\|_{L^{2}}^{2} \cdot \eta^{1 / 2} .
\end{aligned}
$$

If we choose $\epsilon_{1}$ and $\eta$ sufficiently small so that

$$
\eta+\epsilon_{1}+C_{2}\left(\epsilon_{1}\right) \cdot c_{4} \eta^{1 / 2}<\epsilon,
$$

we obtain inequality (2).

\section{Proof of Theorem 1.1}

In this section, we always assume that $(M, g)$ is a smooth topological sphere with bounded Gaussian curvature $K_{g}$. For convenience, we also assume that $\int_{M} d V_{g}=4 \pi$ 
and use the simplified notations as follows:

$$
\nabla:=\nabla_{g}, \quad \Delta:=\Delta_{g}, \quad \int_{M} f:=\int_{M} f d V_{g} .
$$

For any $\epsilon>0$, we define a perturbed (modified also) Liouville energy functional by

$$
I_{\epsilon}(u)=\frac{1}{2} \int_{M}|\nabla u|^{2}+\frac{8 \pi-\epsilon}{4 \pi} \int_{M} K_{g} u-(8 \pi-\epsilon) \ln \int_{M} e^{u},
$$

and denote

$$
E_{\epsilon}=\inf _{u \in H^{1}(M), \int_{M} K_{g} u=0} I_{\epsilon}(u) .
$$

It is easy to see that $I_{\epsilon}(u+C)=I_{\epsilon}(u)$ for any constant $C$. Due to Proposition 2.1 we know that $E_{\epsilon}$ is a finite number. Moreover, we can show

Lemma 3.1 $E_{\epsilon}$ is achieved by a function $u_{\epsilon} \in H^{1}(M)$.

Proof Let $\left\{u_{n}\right\}$ be a minimizing sequence with $\int_{M} K_{g} u_{n}=0$. Choose a small positive number $\epsilon_{1}$ so that

$$
\left(\frac{1}{2}-\epsilon_{1}\right) \cdot \frac{1}{8 \pi-\epsilon}>\frac{1}{16 \pi}+\epsilon_{1} .
$$

Using Proposition 2.1 we have

$$
\begin{aligned}
E_{\epsilon} \leftarrow I_{\epsilon}\left(u_{n}\right) & =\frac{1}{2} \int_{M}\left|\nabla u_{n}\right|^{2}-(8 \pi-\epsilon) \ln \int_{M} e^{u_{n}} \\
& =\left(\frac{1}{2}-\epsilon_{1}\right) \int_{M}\left|\nabla u_{n}\right|^{2}-(8 \pi-\epsilon) \ln \int_{M} e^{u}+\epsilon_{1} \int_{M}\left|\nabla u_{n}\right|^{2} \\
& \geq C\left(\epsilon_{1}\right)+\epsilon_{1} \int_{M}\left|\nabla u_{n}\right|^{2},
\end{aligned}
$$

thus $\left\|\nabla u_{n}\right\|_{L^{2}}<C$. It follows from Lemma 2.2 that $\left\|u_{n}\right\|_{H^{1}}<C$. Further, it implies that $u_{n} \rightarrow u_{\epsilon}$ in $H^{1}(M)$ and $e^{k u_{n}} \rightarrow e^{k u_{\epsilon}}$ in $L^{1}(M)$ for any positive number $k$ by Trudinger's inequality (see, e.g., [20, Theorem A]). It follows that $u_{\epsilon}$ is the minimizer.

Let

$$
v_{\epsilon}=u_{\epsilon}-\ln \int_{M} e^{u_{\epsilon}},
$$

then $v_{\epsilon}$ is also a minimizer, which satisfies the following Euler-Lagrange equation:

$$
-\Delta v_{\epsilon}+\frac{8 \pi-\epsilon}{4 \pi} \cdot K_{g}=(8 \pi-\epsilon) e^{v_{\epsilon}} \quad \text { in } M
$$

and $\int_{M} e^{2 v_{\epsilon}} \leq C$ (since $\left\|v_{\epsilon}\right\|_{H^{1}} \leq C$ ). The standard elliptic estimates yield $v_{\epsilon} \in$ $C^{\alpha}(M)$ for some $\alpha \in(0,1)$. 
To prove Theorem 1.1, we only need to show that $E_{\epsilon}$ is uniformly bounded from below as $\epsilon \rightarrow 0$. We follow closely the similar arguments given early in [15] and [16].

We argue by contradiction. Suppose that $E_{\epsilon}$ is not bounded from below, that is, up to a subsequence (due to the nature of the proof, for convenience we will not distinguish subsequence $\left\{\epsilon_{i}\right\}$ and the original sequence $\{\epsilon\}$ in this section),

$$
\lim _{\epsilon \rightarrow 0} E_{\epsilon}=-\infty
$$

Let $\lambda_{\epsilon}=v_{\epsilon}\left(x_{\epsilon}\right):=\max _{x \in M} v_{\epsilon}(x)$. We first claim

\section{Lemma 3.2}

$$
\lim _{\epsilon \rightarrow 0} \lambda_{\epsilon}=+\infty
$$

Proof Let $v_{\epsilon}^{a}=\frac{1}{4 \pi} \int_{M} v_{\epsilon}$. If $\lambda_{\epsilon}<C$, then $e^{v_{\epsilon}}<C$, thus $\left\|\nabla\left(v_{\epsilon}-v_{\epsilon}^{a}\right)\right\|_{L^{2}}<C$ from Eq. (7). From the Poincaré inequality, we know that $\left\|v_{\epsilon}-v_{\epsilon}^{a}\right\|_{L^{2}}<C$. Since $\| \Delta\left(v_{\epsilon}-\right.$ $\left.v_{\epsilon}^{a}\right) \|_{L^{\infty}} \leq C$, we know from the standard elliptic estimates that $v_{\epsilon}-v_{\epsilon}^{a} \in C^{1}(M)$. If $v_{\epsilon}^{a} \rightarrow-\infty$ is unbounded, we know from Eq. (7) that $v_{\epsilon}-v_{\epsilon}^{a} \rightarrow w$, where $w$ satisfies $\Delta w=2 K_{g}$, this is impossible since $\int_{M} K_{g} \neq 0$; If $v_{\epsilon}^{a}$ is bounded, then $v_{\epsilon}=$ $\left(v_{\epsilon}-v_{\epsilon}^{a}\right)+v_{\epsilon}^{a}$ is bounded, thus $E_{\epsilon}$ is bounded from below, this is a contradiction to (8).

Assume that, up to a subsequence, $x_{\epsilon} \rightarrow \bar{x} \in M$. In a neighborhood of $\bar{x}$, we choose a normal coordinate system, and define

$$
\varphi_{\epsilon}(x)=v_{\epsilon}\left(\tau_{\epsilon}^{-1} x+x_{\epsilon}\right)-2 \ln \tau_{\epsilon},
$$

where $\tau_{\epsilon}:=e^{\lambda_{\epsilon} / 2}$, and without confusion, we use $\tau_{\epsilon}^{-1} x+x_{\epsilon}$ to represent $\exp _{x_{\epsilon}}\left(\tau_{\epsilon}^{-1} x\right)$. For any given $R>0$, if $\epsilon$ is sufficiently small, $\varphi_{\epsilon}$ satisfies

$$
-\Delta \varphi_{\epsilon}+\frac{8 \pi-\epsilon}{4 \pi \tau_{\epsilon}^{2}} \cdot K_{g}=(8 \pi-\epsilon) e^{\varphi_{\epsilon}} \quad \text { in } B_{2 R}(0) \subset R^{2} .
$$

The behavior of $v_{\epsilon}$ in a tiny neighborhood of $\bar{x}$ can be described by the behavior of $\varphi_{\epsilon}$ in a large ball $B_{2 R}(0) \subset R^{2}$.

Lemma 3.3 For a fixed $R>0$, there is a constant $C(R)$ such that

$$
\left|\varphi_{\epsilon}(x)\right|<C(R) \quad \forall x \in B_{R}(0) .
$$

Proof Let $\varphi_{\epsilon}^{(1)}$ be the unique solution to

$$
\begin{cases}-\Delta \varphi_{\epsilon}^{(1)}+\frac{8 \pi-\epsilon}{4 \pi \tau_{\epsilon}^{2}} \cdot K_{g}=(8 \pi-\epsilon) e^{\varphi_{\epsilon}} & \text { in } B_{2 R}(0), \\ \varphi_{\epsilon}^{(1)}=0 & \text { on } \partial B_{2 R}(0) .\end{cases}
$$

Since $e^{\varphi_{\epsilon}} \leq 1$, we know that $\left|\varphi_{\epsilon}^{(1)}\right|<C_{1}(R)$ for $x \in B_{R}(0)$. Let $\varphi_{\epsilon}^{(2)}=\varphi_{\epsilon}-\varphi_{\epsilon}^{(1)}$. Then $\varphi_{\epsilon}^{(2)}$ is a harmonic function bounded above by $C_{1}(R)$. Applying the Harnack 
inequality to $2 C_{1}(R)+\varphi_{\epsilon}^{(2)}$ we have

$$
\frac{1}{C} \leq \frac{2 C_{1}(R)+\varphi_{\epsilon}^{(2)}(x)}{2 C_{1}(R)+\varphi_{\epsilon}^{(2)}(0)}<C \quad \forall x \in B_{R}(0) .
$$

This and the bound of $\varphi_{\epsilon}^{(1)}$ yield Lemma 3.3.

Since $\varphi_{\epsilon}$ is uniformly bounded in any fixed ball, we know, based on the standard elliptic estimates, that

$$
\varphi_{\epsilon} \rightarrow \varphi_{0} \quad \text { in } C^{1, \alpha}\left(B_{R}(0)\right),
$$

for some $\alpha \in(0,1)$, where $\varphi_{0}$ satisfies

$$
-\Delta \varphi_{0}=8 \pi e^{\varphi_{0}} \quad \text { in } R^{2} .
$$

On the other hand, we note that

$$
\varphi_{0}(x) \leq \varphi_{0}(0)=1 \quad \text { and } \quad \int_{R^{2}} e^{\varphi_{0}} \leq \lim _{R \rightarrow+\infty}\left(\varlimsup_{\epsilon \rightarrow 0} \int_{B_{R}} e^{\varphi_{\epsilon}}\right) \leq 1 .
$$

From the classification of constant curvature metric on $S^{2}$ or the PDE's result of Chen and $\mathrm{Li}[12]$ we know that

$$
\varphi_{0}(x)=2 \ln \frac{1}{1+\pi|x|^{2}} .
$$

Away from the singular point $\bar{x}$, we have the following global estimate.

Lemma 3.4 For any compact domain $K \subset \subset M \backslash\{\bar{x}\}$, there is a constant $C(K)$ such that

$$
\left\|v_{\epsilon}-\frac{1}{4 \pi} \int_{M} v_{\epsilon}\right\|_{L^{\infty}(K)}<C(K) .
$$

The proof of Lemma 3.4 is based on a lemma due to Brezis and Merle [5]. Such a lemma plays the same role as the sharp Sobolev inequalities play in higher dimensional cases. To keep our argument intact, we relegate the proof of this lemma to the end of this section.

Note that for any $1 \leq q<2$,

$$
\left\|\nabla v_{\epsilon}\right\|_{L^{q}(M)}<C_{q} .
$$

This is because for any $\varphi \in W^{1, q /(q-1)}(M)$ with $\int_{M} \varphi=0$ and $\|\varphi\|_{W^{1, q /(q-1)}}=1$ (thus $\left.\varphi \in L^{\infty}(M)\right)$,

$$
\left|\int_{M} \nabla v_{\epsilon} \nabla \varphi\right|=\left|\int_{M} \Delta v_{\epsilon} \varphi\right| \leq C
$$

We derive from the above and (8) that

$$
4 \pi v_{\epsilon}^{a}=\int_{M} K_{g} v_{\epsilon}-\int_{M} K_{g}\left(v_{\epsilon}-v_{\epsilon}^{a}\right)
$$




$$
\leq \int_{M} K_{g} v_{\epsilon}-C \rightarrow-\infty
$$

From Lemma 3.4 and (15), we conclude, via the standard elliptic estimates, that

$$
v_{\epsilon}-v_{\epsilon}^{a} \rightarrow G(x, \bar{x}) \quad \text { in } C^{1, \alpha}(K)
$$

for any compact domain $K$ in $M \backslash\{\bar{x}\}$, where $G(x, \bar{x})$ is the Green function in $M$ satisfying

$$
\left\{\begin{array}{l}
-\Delta G+2 K_{g}=8 \pi \delta_{\bar{x}} \text { in } M, \\
\int_{M} G=0 .
\end{array}\right.
$$

Since $K_{g}$ is bounded, in a normal coordinate system centered at $\bar{x}$,

$$
G(x, \bar{x})=-4 \ln |x|+A(\bar{x})+o(1),
$$

where $A(\bar{x})$ is a constant depending on the location of $\bar{x}, o(1) \rightarrow 0$ as $|x| \rightarrow 0$.

To complete the proof of the main theorem, we also need a lower bound for $v_{\epsilon}$ away from the singular point $\bar{x}$.

Lemma 3.5 For any fixed $R>0$, let $r_{\epsilon}=\tau_{\epsilon}^{-1} R$. Then

$$
v_{\epsilon}(x) \geq G\left(x, x_{\epsilon}\right)-\lambda_{\epsilon}-2 \ln \frac{1+\pi R^{2}}{R^{2}}-A(\bar{x})+o_{\epsilon}(1) \quad \forall x \in M \backslash B\left(x_{\epsilon}, r_{\epsilon}\right),
$$

where $o_{\epsilon}(1) \rightarrow 0$ as $\epsilon \rightarrow 0$.

Proof On $\partial B\left(x_{\epsilon}, r_{\epsilon}\right)$, we define $C_{\epsilon}^{*}:=\left.\left(v_{\epsilon}-G\left(x, x_{\epsilon}\right)\right)\right|_{|x|=r_{\epsilon}}$. From (10) and (13) we know that

$$
C_{\epsilon}^{*}=-\lambda_{\epsilon}-2 \ln \frac{1+\pi R^{2}}{R^{2}}-A(\bar{x})+o_{\epsilon}(1)
$$

Let

$$
\tilde{K}_{g}(x)= \begin{cases}K_{g}(x), & \text { if } K_{g}(x) \leq 0, \\ \phi(x) K_{g}(x), & \text { if } K_{g}(x)>0,\end{cases}
$$

where $0 \leq \phi \leq 1$ is a measurable function such that $\int_{M} \tilde{K}_{g}=0$. Let $h(x)$ be one solution to $\Delta h=\tilde{K}_{g}$ in $M$ (which is bounded). We consider $v_{\epsilon}(x)-G\left(x, x_{\epsilon}\right)-C_{\epsilon}^{*}+$ $\frac{\epsilon}{4 \pi} h(x)$ in $M \backslash B\left(x_{\epsilon}, r_{\epsilon}\right)$. Since $-\Delta\left(v_{\epsilon}(x)-G\left(x, x_{\epsilon}\right)-C_{\epsilon}^{*}+\frac{\epsilon}{4 \pi} h(x)\right) \geq \frac{\epsilon\left(K_{g}-\tilde{K}_{g}\right)}{4 \pi} \geq$ 0 , we have (19) via the maximum principle.

We are now ready to complete the proof of the main theorem.

We need to estimate $E_{\epsilon}=I_{\epsilon}\left(v_{\epsilon}\right)$. For any fixed small $\delta>0$, we assume that $\epsilon$ is sufficiently small so that $\delta>r_{\epsilon}$. Then

$$
\begin{aligned}
\int_{M}\left|\nabla v_{\epsilon}\right|^{2} & =\int_{M \backslash B\left(x_{\epsilon}, \delta\right)}\left|\nabla v_{\epsilon}\right|^{2}+\int_{B\left(x_{\epsilon}, \delta\right) \backslash B\left(x_{\epsilon}, r_{\epsilon}\right)}\left|\nabla v_{\epsilon}\right|^{2}+\int_{B\left(x_{\epsilon}, r_{\epsilon}\right)}\left|\nabla v_{\epsilon}\right|^{2} \\
& :=\mathbf{I}_{1}+\mathbf{I}_{2}+\mathbf{I}_{3} .
\end{aligned}
$$


First of all, it is easy to see from (10) and (13) that

$$
\begin{aligned}
\mathbf{I}_{3} & =\int_{B\left(x_{\epsilon}, r_{\epsilon}\right)}\left|\nabla \varphi_{0}\right|^{2} d x+o_{\epsilon}(1)=\int_{B(R)}\left|\nabla\left(2 \ln \frac{1}{1+\pi|x|^{2}}\right)\right|^{2} d x+o_{\epsilon}(1) \\
& =32 \pi^{3} \int_{0}^{R} \frac{r^{2} r}{\left(1+\pi r^{2}\right)^{2}} d r+o_{\epsilon}(1) \\
& =16 \pi\left(\ln \left(1+\pi R^{2}\right)-1\right)+o_{\epsilon}(1)+o_{R}(1),
\end{aligned}
$$

where $o_{R}(1) \rightarrow 0$ as $R \rightarrow \infty$.

Since $v_{\epsilon}(x)-v_{\epsilon}^{a} \rightarrow G\left(x, x_{\epsilon}\right)$ in $C_{\text {loc }}^{2}\left(M \backslash\left\{x_{\epsilon}\right\}\right)$, we obtain

$$
\begin{aligned}
\mathbf{I}_{1}=\int_{M \backslash B\left(x_{\epsilon}, \delta\right)}\left|\nabla v_{\epsilon}\right|^{2} & =\int_{M \backslash B\left(x_{\epsilon}, \delta\right)}|\nabla G|^{2}+o_{\epsilon}(1) \\
& =-\int_{M \backslash B\left(x_{\epsilon}, \delta\right)} 2 K_{g} G-\int_{\partial B\left(x_{\epsilon}, \delta\right)} G \frac{\partial G}{\partial \mathbf{n}}+o_{\epsilon}(1) .
\end{aligned}
$$

To estimate $\mathbf{I}_{2}$, we first use Eq. (7) to get

$$
\begin{aligned}
\mathbf{I}_{2}= & -\int_{B\left(x_{\epsilon}, \delta\right) \backslash B\left(x_{\epsilon}, r_{\epsilon}\right)} \Delta v_{\epsilon} \cdot v_{\epsilon}+\int_{\partial\left(B\left(x_{\epsilon}, \delta\right) \backslash B\left(x_{\epsilon}, r_{\epsilon}\right)\right)} v_{\epsilon} \frac{\partial v_{\epsilon}}{\partial \mathbf{n}} \\
= & \int_{B\left(x_{\epsilon}, \delta\right) \backslash B\left(x_{\epsilon}, r_{\epsilon}\right)}\left(-\frac{8 \pi-\epsilon}{4 \pi} K_{g} \cdot v_{\epsilon}+(8 \pi-\epsilon) v_{\epsilon} e^{v_{\epsilon}}\right) \\
& +\int_{\partial\left(B\left(x_{\epsilon}, \delta\right) \backslash B\left(x_{\epsilon}, r_{\epsilon}\right)\right)} v_{\epsilon} \frac{\partial v_{\epsilon}}{\partial \mathbf{n}} .
\end{aligned}
$$

Applying Lemma 3.5, we have

$$
\begin{aligned}
\int_{B\left(x_{\epsilon}, \delta\right) \backslash B\left(x_{\epsilon}, r_{\epsilon}\right)}(8 \pi-\epsilon) v_{\epsilon} e^{v_{\epsilon}} \geq & -\lambda_{\epsilon} \cdot(8 \pi-\epsilon) \int_{B\left(x_{\epsilon}, \delta\right) \backslash B\left(x_{\epsilon}, r_{\epsilon}\right)} e^{v_{\epsilon}} \\
& +\int_{B\left(x_{\epsilon}, \delta\right) \backslash B\left(x_{\epsilon}, r_{\epsilon}\right)}(8 \pi-\epsilon) G e^{v_{\epsilon}}+o_{\epsilon}(1)+o_{R}(1),
\end{aligned}
$$

where $o_{\epsilon}(1) \rightarrow 0$ as $\epsilon \rightarrow 0$ and $o_{R}(1) \rightarrow 0$ as $R \rightarrow+\infty$. From Eqs. (7), (16) and (17) we obtain

$$
\begin{aligned}
\int_{B\left(x_{\epsilon}, \delta\right) \backslash B\left(x_{\epsilon}, r_{\epsilon}\right)}(8 \pi-\epsilon) G e^{v_{\epsilon}}= & -\int_{B\left(x_{\epsilon}, \delta\right) \backslash B\left(x_{\epsilon}, r_{\epsilon}\right)} 2 K_{g} v_{\epsilon} \\
& +\int_{B\left(x_{\epsilon}, \delta\right) \backslash B\left(x_{\epsilon}, r_{\epsilon}\right)} \frac{8 \pi-\epsilon}{4 \pi} K_{g} \cdot G \\
& +\int_{\partial\left(B\left(x_{\epsilon}, \delta\right) \backslash B\left(x_{\epsilon}, r_{\epsilon}\right)\right)}\left(v_{\epsilon} \frac{\partial G}{\partial \mathbf{n}}-G \frac{\partial v_{\epsilon}}{\partial \mathbf{n}}\right) \\
= & -\int_{B\left(x_{\epsilon}, \delta\right) \backslash B\left(x_{\epsilon}, r_{\epsilon}\right)} 2 K_{g} v_{\epsilon}+\int_{\partial B\left(x_{\epsilon}, \delta\right)} v_{\epsilon}^{a} \frac{\partial G}{\partial \mathbf{n}}
\end{aligned}
$$




$$
\begin{aligned}
& -\int_{\partial B\left(x_{\epsilon}, r_{\epsilon}\right)}\left(v_{\epsilon} \frac{\partial G}{\partial \mathbf{n}}-G \frac{\partial v_{\epsilon}}{\partial \mathbf{n}}\right) \\
& +o_{\epsilon}(1)+o_{\delta}(1)
\end{aligned}
$$

where $o_{\delta}(1) \rightarrow 0$ as $\delta \rightarrow 0$, and

$$
\begin{aligned}
-\lambda_{\epsilon} \cdot(8 \pi-\epsilon) \int_{B\left(x_{\epsilon}, \delta\right) \backslash B\left(x_{\epsilon}, r_{\epsilon}\right)} e^{v_{\epsilon}}= & -\lambda_{\epsilon} \cdot \int_{B\left(x_{\epsilon}, \delta\right) \backslash B\left(x_{\epsilon}, r_{\epsilon}\right)} \frac{8 \pi-\epsilon}{4 \pi} \cdot K_{g} \\
& +\lambda_{\epsilon} \cdot \int_{\partial\left(B\left(x_{\epsilon}, \delta\right) \backslash B\left(x_{\epsilon}, r_{\epsilon}\right)\right)} \frac{\partial v_{\epsilon}}{\partial \mathbf{n}} .
\end{aligned}
$$

Thus

$$
\begin{aligned}
\mathbf{I}_{2} \geq & -\frac{16 \pi-\epsilon}{4 \pi} \cdot \int_{B\left(x_{\epsilon}, \delta\right) \backslash B\left(x_{\epsilon}, r_{\epsilon}\right)} K_{g} v_{\epsilon}+\int_{\partial\left(B\left(x_{\epsilon}, \delta\right) \backslash B\left(x_{\epsilon}, r_{\epsilon}\right)\right)} v_{\epsilon} \frac{\partial v_{\epsilon}}{\partial \mathbf{n}} \\
& +\int_{\partial B\left(x_{\epsilon}, \delta\right)} v_{\epsilon}^{a} \frac{\partial G}{\partial \mathbf{n}}-\int_{\partial B\left(x_{\epsilon}, r_{\epsilon}\right)}\left(v_{\epsilon} \frac{\partial G}{\partial \mathbf{n}}-G \frac{\partial v_{\epsilon}}{\partial \mathbf{n}}\right) \\
& -\lambda_{\epsilon} \cdot \int_{B\left(x_{\epsilon}, \delta\right) \backslash B\left(x_{\epsilon}, r_{\epsilon}\right)} \frac{8 \pi-\epsilon}{4 \pi} \cdot K_{g}+\lambda_{\epsilon} \cdot \int_{\partial\left(B\left(x_{\epsilon}, \delta\right) \backslash B\left(x_{\epsilon}, r_{\epsilon}\right)\right)} \frac{\partial v_{\epsilon}}{\partial \mathbf{n}} \\
& +o_{\epsilon}(1)+o_{\delta}(1)+o_{R}(1) \\
= & -\frac{16 \pi-\epsilon}{4 \pi} \cdot \int_{B\left(x_{\epsilon}, \delta\right) \backslash B\left(x_{\epsilon}, r_{\epsilon}\right)} K_{g} v_{\epsilon}-\lambda_{\epsilon} \cdot \int_{B\left(x_{\epsilon}, \delta\right) \backslash B\left(x_{\epsilon}, r_{\epsilon}\right)} \frac{8 \pi-\epsilon}{4 \pi} \cdot K_{g} \\
& +o_{\epsilon}(1)+o_{\delta}(1)+o_{R}(1) \\
& -\int_{\partial B\left(x_{\epsilon}, r_{\epsilon}\right)} \frac{\partial v_{\epsilon}}{\partial \mathbf{n}} \cdot\left(v_{\epsilon}-\left(G-\lambda_{\epsilon}\right)\right)-\int_{\partial B\left(x_{\epsilon}, r_{\epsilon}\right)} v_{\epsilon} \frac{\partial G}{\partial \mathbf{n}} \\
& +\int_{\partial B\left(x_{\epsilon}, \delta\right)} v_{\epsilon} \cdot \frac{\partial v_{\epsilon}}{\partial \mathbf{n}}+v_{\epsilon}^{a} \cdot \int_{\partial B\left(x_{\epsilon}, \delta\right)} \frac{\partial G}{\partial \mathbf{n}}+\lambda_{\epsilon} \cdot \int_{\partial B\left(x_{\epsilon}, \delta\right)} \frac{\partial v_{\epsilon}}{\partial \mathbf{n}} .
\end{aligned}
$$

We now estimate the boundary term in the right hand side of the above inequality. From (10), (13) and Lemma 3.5, we know that

$$
-\int_{\partial B\left(x_{\epsilon}, r_{\epsilon}\right)} \frac{\partial v_{\epsilon}}{\partial \mathbf{n}} \cdot\left(v_{\epsilon}-\left(G-\lambda_{\epsilon}\right)\right) \geq \frac{8 \pi^{2} R^{2}}{1+\pi R^{2}} \cdot\left(-A(\bar{x})-2 \ln \frac{1+\pi R^{2}}{R^{2}}\right)+o_{\epsilon}(1),
$$

and

$$
\begin{aligned}
& -\int_{\partial B\left(x_{\epsilon}, r_{\epsilon}\right)} v_{\epsilon} \frac{\partial G}{\partial \mathbf{n}} \\
& =-\lambda_{\epsilon} \int_{\partial B\left(x_{\epsilon}, r_{\epsilon}\right)} \frac{\partial G}{\partial \mathbf{n}}-16 \pi \ln \left(1+\pi R^{2}\right)+o_{\epsilon}(1)+o_{R}(1) \\
& =\lambda_{\epsilon} \int_{M \backslash B\left(x_{\epsilon}, r_{\epsilon}\right)} \Delta G-16 \pi \ln \left(1+\pi R^{2}\right)+o_{\epsilon}(1)+o_{R}(1)
\end{aligned}
$$




$$
=2 \lambda_{\epsilon} \int_{M \backslash B\left(x_{\epsilon}, r_{\epsilon}\right)} K_{g}-16 \pi \ln \left(1+\pi R^{2}\right)+o_{\epsilon}(1)+o_{R}(1) .
$$

From Eq. (7) we have

$$
\begin{aligned}
\lambda_{\epsilon} \cdot \int_{\partial B\left(x_{\epsilon}, \delta\right)} \frac{\partial v_{\epsilon}}{\partial \mathbf{n}} & =-\lambda_{\epsilon} \cdot \int_{M \backslash B\left(x_{\epsilon}, \delta\right)} \Delta v_{\epsilon} \\
& =-\frac{8 \pi-\epsilon}{4 \pi} \lambda_{\epsilon} \int_{M \backslash B\left(x_{\epsilon}, \delta\right)} K_{g}+\lambda_{\epsilon} \int_{M \backslash B\left(x_{\epsilon}, \delta\right)}(8 \pi-\epsilon) e^{v_{\epsilon}} \\
& \geq-\frac{8 \pi-\epsilon}{4 \pi} \lambda_{\epsilon} \int_{M \backslash B\left(x_{\epsilon}, \delta\right)} K_{g} .
\end{aligned}
$$

Similarly, we have

$$
v_{\epsilon}^{a} \cdot \int_{\partial B\left(x_{\epsilon}, \delta\right)} \frac{\partial G}{\partial \mathbf{n}}=-2 v_{\epsilon}^{a} \int_{M \backslash B\left(x_{\epsilon}, \delta\right)} K_{g}
$$

and

$$
\begin{aligned}
v_{\epsilon}^{a} \cdot \int_{\partial B\left(x_{\epsilon}, \delta\right)} \frac{\partial v_{\epsilon}}{\partial \mathbf{n}} & =-\frac{8 \pi-\epsilon}{4 \pi} v_{\epsilon}^{a} \int_{M \backslash B\left(x_{\epsilon}, \delta\right)} K_{g}+v_{\epsilon}^{a} \int_{M \backslash B\left(x_{\epsilon}, \delta\right)}(8 \pi-\epsilon) e^{v_{\epsilon}} \\
& =-\frac{8 \pi-\epsilon}{4 \pi} v_{\epsilon}^{a} \int_{M \backslash B\left(x_{\epsilon}, \delta\right)} K_{g}+v_{\epsilon}^{a} e^{v_{\epsilon}^{a}} \int_{M \backslash B\left(x_{\epsilon}, \delta\right)}(8 \pi-\epsilon) e^{v_{\epsilon}-v_{\epsilon}^{a}} \\
& =-\frac{8 \pi-\epsilon}{4 \pi} v_{\epsilon}^{a} \int_{M \backslash B\left(x_{\epsilon}, \delta\right)} K_{g}+o_{\epsilon}(1) .
\end{aligned}
$$

For the interior terms, we note that

$$
\begin{aligned}
\int_{B\left(x_{\epsilon}, \delta\right) \backslash B\left(x_{\epsilon}, r_{\epsilon}\right)} K_{g} v_{\epsilon} & =v_{\epsilon}^{a} \int_{B\left(x_{\epsilon}, \delta\right) \backslash B\left(x_{\epsilon}, r_{\epsilon}\right)} K_{g}+\int_{B\left(x_{\epsilon}, \delta\right) \backslash B\left(x_{\epsilon}, r_{\epsilon}\right)} K_{g}\left(v_{\epsilon}-v_{\epsilon}^{a}\right) \\
& =v_{\epsilon}^{a} \int_{B\left(x_{\epsilon}, \delta\right) \backslash B\left(x_{\epsilon}, r_{\epsilon}\right)} K_{g}+o_{\delta}(1),
\end{aligned}
$$

where we use the Poincaré inequality and (14).

We conclude

$$
\begin{aligned}
\mathbf{I}_{2} \geq & -(16 \pi-\epsilon) v_{\epsilon}^{a}+\frac{16 \pi-\epsilon}{4 \pi} \int_{B\left(x_{\epsilon}, r_{\epsilon}\right)} K_{g} \cdot v_{\epsilon}^{a} \\
& -8 \pi A(\bar{x})-16 \pi \ln \pi-16 \pi \ln \left(1+\pi R^{2}\right) \\
& +\epsilon \lambda_{\epsilon} \cdot\left(1-\frac{1}{4 \pi} \int_{B\left(x_{\epsilon}, r_{\epsilon}\right)} K_{g}\right) \\
& +\int_{\partial B\left(x_{\epsilon}, \delta\right)} G \frac{\partial G}{\partial \mathbf{n}} \\
& +o_{\epsilon}(1)+o_{\delta}(1)+o_{R}(1) .
\end{aligned}
$$


We have, from (20)-(22),

$$
\begin{aligned}
I_{\epsilon}\left(v_{\epsilon}\right) \geq & -4 \pi A(\bar{x})-8 \pi \ln \pi-8 \pi \\
& +\epsilon \lambda_{\epsilon} \cdot\left(\frac{1}{2}-\frac{1}{8 \pi} \int_{B\left(x_{\epsilon}, r_{\epsilon}\right)} K_{g}\right) \\
& -\frac{1}{2} \epsilon v_{\epsilon}^{a}+\frac{16 \pi-\epsilon}{8 \pi} \int_{B\left(x_{\epsilon}, r_{\epsilon}\right)} K_{g} \cdot v_{\epsilon}^{a} \\
& -\int_{M \backslash B\left(x_{\epsilon}, \delta\right)} K_{g} G+\frac{8 \pi-\epsilon}{4 \pi} \int_{M} K_{g} \cdot\left(v_{\epsilon}-v_{\epsilon}^{a}\right) \\
& +o_{\epsilon}(1)+o_{\delta}(1)+o_{R}(1) .
\end{aligned}
$$

From (15), we know that $v_{\epsilon}^{a} \rightarrow-\infty$. Also, since $\lambda_{\epsilon} \cdot \operatorname{vol}\left(B\left(x_{\epsilon}, r_{\epsilon}\right)\right)=o_{\epsilon}(1)$, we know from Lemma 3.5 that $v_{\epsilon}^{a} \cdot \operatorname{vol}\left(B\left(x_{\epsilon}, r_{\epsilon}\right)\right)=o_{\epsilon}(1)$. We finally have

$$
\lim _{\epsilon \rightarrow 0} E_{\epsilon} \geq-4 \pi A(\bar{x})-8 \pi \ln \pi-8 \pi+C
$$

for some constant $C$. Thus Theorem 1.1 is proved.

We are left to prove Lemma 3.4. We need the following lemma due to Brezis and Merle [5].

Lemma 3.6 Let $\Omega \subset M$ be a bounded domain and $u$ be a solution to the following equation:

$$
\begin{cases}-\Delta u=f(x) & \text { in } \Omega, \\ u=0 & \text { on } \partial \Omega .\end{cases}
$$

If $f(x) \in L^{1}(\Omega)$, then for any $0<\delta<4 \pi$, there is a constant $C(\delta)$ such that

$$
\int_{\Omega} \exp \left\{\frac{(4 \pi-\delta)|u(x)|}{\|f\|_{L^{1}(\Omega)}}\right\} \leq C(\delta) .
$$

Lemma 3.6 was originally proved for a bounded domain in $R^{2}$, but it is easy to see that the same result holds on any domain in a compact Riemann surface. We now return to the

Proof of Lemma 3.4 Let $K \subset \subset M \backslash\{\bar{x}\}$. We choose other two compact sets $K_{1}$ and $K_{2}$ in $M \backslash\{\bar{x}\}$ such that $K \subset \subset K_{1} \subset \subset K_{2} \subset \subset M \backslash\{\bar{x}\}$. It follows from (10) that

$$
\lim _{\epsilon \rightarrow 0} \int_{K_{2}} e^{v_{\epsilon}}=0 .
$$

Let $v_{\epsilon}^{1}$ be the unique solution to

$$
\begin{cases}-\Delta v_{\epsilon}^{1}=(8 \pi-\epsilon) e^{v_{\epsilon}} & \text { on } K_{2}, \\ v_{\epsilon}^{1}=0 & \text { on } \partial K_{2},\end{cases}
$$


and $v_{\epsilon}^{2}=v_{\epsilon}-v_{\epsilon}^{1}-v_{\epsilon}^{a}$. From (23) and Lemma 3.6 we know that $e^{\left|v_{\epsilon}^{1}\right|} \in L^{p}\left(K_{2}\right)$ for some $1<p<2$; thus $\left\|v_{\epsilon}^{1}\right\|_{L^{p}\left(K_{2}\right)} \leq C$. On the other hand, we note $v_{\epsilon}^{2}$ satisfying $\Delta v_{\epsilon}^{2}=\frac{8 \pi-\epsilon}{4 \pi} K_{g}$ in $K_{2}$, we have, from the interior $L^{p}$ estimates, for $K_{1} \subset \subset K_{2}$,

$$
\begin{aligned}
\left\|v_{\epsilon}^{2}\right\|_{L^{\infty}\left(K_{1}\right)} & \leq C\left\|v_{\epsilon}^{2}\right\|_{L^{p}\left(K_{2}\right)} \\
& \leq C\left(\left\|v_{\epsilon}-v_{\epsilon}^{a}\right\|_{L^{p}\left(K_{2}\right)}+\left\|v_{\epsilon}^{1}\right\|_{L^{p}\left(K_{2}\right)}\right) \\
& \leq C\left(\left\|\nabla v_{\epsilon}\right\|_{L^{p}\left(K_{2}\right)}+\left\|v_{\epsilon}^{1}\right\|_{L^{p}\left(K_{2}\right)}\right) \\
& \leq C,
\end{aligned}
$$

where we also use (14). In turn, we know that $\int_{K_{1}} e^{p v_{\epsilon}^{1}} \cdot e^{p v_{\epsilon}^{2}} \leq C$. Combining with (15) we have

$$
\begin{aligned}
\int_{K_{1}} e^{p v_{\epsilon}} & \leq \int_{K_{1}} e^{p v_{\epsilon}^{a}} \cdot e^{p v_{\epsilon}^{1}} \cdot e^{p v_{\epsilon}^{2}} \\
& \leq C,
\end{aligned}
$$

we then know from (24) that $\left\|v_{\epsilon}^{1}\right\|_{L^{\infty}(K)} \leq C$, thus $\left\|v_{\epsilon}-v_{\epsilon}^{a}\right\|_{L^{\infty}(K)} \leq C$.

Acknowledgements We thank Yuxiang Li for pointing out the proof of Lemma 3.5 in an early version is incomplete. We also thank the referee for the careful reviewing and comments.

\section{References}

1. Aubin, T.: Problèmes isopérimétriques et espaces de Sobolev. J. Differ. Geom. 11, 573-598 (1976)

2. Aubin, T.: Meilleures constantes dans le thorme d'inclusion de Sobolev et un thorme de Fredholm non linaire pour la transformation conforme de la courbure scalaire. J. Funct. Anal. 32(2), 148-174 (1979) (French)

3. Bando, S., Mabuchi, T.: Uniqueness of Einstein Kähler metrics modulo connected group actions. In: Algebraic Geometry, Sendai, 1985. Adv. Stud. Pure Math., vol. 10, pp. 11-40. North-Holland, Amsterdam (1987)

4. Berger, M.: Riemannian structures of prescribed Gaussian curvature for compact 2-manifolds. J. Differ. Geom. 5, 325-332 (1971)

5. Brezis, H., Merle, F.: Uniform estimates and blow-up behavior for solutions of $-\Delta u=V(x) e^{u}$ in two dimensions. Commun. Partial Differ. Equ. 16(8-9), 1223-1253 (1991)

6. Calabi, E.: Extremal Kähler metrics. In: Seminar on Differential Geometry. Ann. of Math. Studies, vol. 16, pp. 259-290. Princeton University Press, Princeton (1982)

7. Chen, X.X.: Calabi flow in Riemann surfaces revisited: a new point of view. Int. Math. Res. Not. 6, 275-297 (2001)

8. Chen, X.X.: Lower bound of the energy functional $E_{1}$ (I)—stability of Kähler Ricci flow. J. Geom. Anal. 16(1), 23-28 (2006)

9. Chen, X.X., Tian, G.: Ricci flow on Kähler-Einstein surfaces. Invent. Math. 147(3), 487-544 (2002). doi:10.1007/s002220100181

10. Chen, X.X., Tian, G.: Geometry of Kähler metrics and foliations by holomorphic discs. Publ. Math. JHÉS 107, 1-107 (2008)

11. Chen, X.X., Lu, P., Tian, G.: A note on uniformization of Riemann surfaces by Ricci flow. Proc. Am. Math. Soc. 134(11), 3391-3393 (2006)

12. Chen, W., Li, C.: Classification of solutions of some nonlinear elliptic equations. Duke Math. J. 63(3), 615-622 (1991) 
13. Cherrier, P.: Une ingalit de Sobolev sur les varits riemanniennes. Bull. Sci. Math. 103(4), 353-374 (1979) (French)

14. Chow, B.: The Ricci flow on the 2-sphere. J. Differ. Geom. 33(2), 325-334 (1991)

15. Ding, W., Jost, J., Li, J., Wang, G.: The differential equation $\delta u=8 \pi-8 \pi h e^{u}$ on a compact Riemann surface. Asian J. Math. 1(2), 230-248 (1997)

16. Ding, W., Jost, J., Li, J., Wang, G.: An analysis of the two-vortex case in the Chern-Simons Higgs model. Calc. Var. Partial Differ. Equ. 7(1), 87-97 (1998)

17. Hamilton, R.: The Ricci flow on surfaces. In: Mathematics and General Relativity, Santa Cruz, CA, 1986. Contemp. Math., vol. 71, pp. 237-262. Am. Math. Soc., Providence (1988)

18. Hebey, E., Vaugon, M.: Meilleures constantes dans le theoreme d'inclusion de Sobolev. Ann. Inst. Henri Poincaré, Anal. Non Linéaire 13, 57-93 (1996)

19. Li, Y.Y., Zhu, M.: Sharp Sobolev trace inequality on Riemannian manifolds with boundary. Commun. Pure Appl. Math. 50, 449-487 (1997)

20. Li, J., Zhu, M.: Sharp local embedding inequalities. Commun. Pure Appl. Math. 59, 122-144 (2006)

21. Li, S., Zhu, M.: A sharp inequality and its applications. Commun. Contemp. Math. 11(3), 433-446 (2009)

22. Pali, N.: A consequence of a lower bound of the K-energy. Int. Math. Res. Not. 2005(50), 3081-3090 (2005)

23. Osgood, B., Phillips, R., Sarnak, P.: Compact isospectral sets of surfaces. J. Funct. Anal. 80(1), 212234 (1988)

24. Song, J., Weinkove, B.: Energy functionals and canonical Kähler metrics. Duke Math. J. 137(1), 159184 (2007)

25. Tosatti, V.: On the critical points of the $E_{k}$ functionals in Kähler geometry. Proc. Am. Math. Soc. 135(12), 3985-3988 (2007) 